\title{
Making a financial Time Machine: A Multi- touch application to enable interactive 3-D visualization of distant savings goals
}

\author{
K. C. Scott-Brown* \\ R. Henderson \\ Whitespace Research Group* \\ University of Abertay Dundee \\ Bell St, Dundee \\ k.scott-brown@abertay.ac.uk
}

\author{
O. Ahrani Dahrani \\ D. Cernagovs \\ J. Turner \\ University of Abertay Dundee \\ Bell St, Dundee
}

\author{
H. Mclaughlin \\ H. Tarbert** \\ Dundee Business School** \\ University of Abertay Dundee \\ Bell St, Dundee
}

j.turner@abertay.ac.uk, h.tarbert@abertay.ac.uk

\begin{abstract}
Financial planning and decision making for the general public continues to vex and perplex in equal measure. Whilst the tools presented by a typical desktop computer should make the task easier, the recent financial crisis confirms the increasing difficulty that people have in calculating the benefits of deferring consumption for future gains (i.e. Saving). We present an interactive concept demonstration for Microsoft SurfaceTM that tackles two of the key barriers to saving decision making. Firstly we show an interface that avoid the laborious writing down or inputting of data and instead embodies the cognitive decision of allocation of resources in a physical gesture based interface, where the scale of the investment or expenditure correlates with the scale of the gesture. Second we show how a fast-forward based animation can demonstrate the impact of small increments in savings to a long term savings goal in a strategy game-based, interactive format. The platform uses custom software (XNATM format) as opposed to the more usual WPFTM format found on Surface applications. This enables dynamic 3-D graphical icons to be used to maximize the interactive appeal of the interface. Demonstration and test trial feedback indicates that this platform can be adapted to suit the narrative of individual purchasing decisions to inform educate diverse user groups about the long term consequences of small financial decisionsEye-tracking, awareness tools, machine learning, coordination, expertise
\end{abstract}

Artistic and aesthetic aspects of interaction and interface design, Computer games design and development, Information visualization and presentation, Innovative interface design, Interface agents and interface characters, Multimodal interfaces, New interaction techniques and devices, Play and the use of play, Microsoft Surface, XNA ${ }^{T M}$, WPF ${ }^{T M}$.

\section{INTRODUCTION}

Buy today, or save for a rainy day? The fundamental debate of the average student saver; this question underlies a fundamental error of human reasoning known as discounting of future gains $[1,2,3]$. The average human will behave in an apparently irrational way when presented with scare resources and a series of choices for allocation. In general experiments or studies of economic or hedonic choices, participants are likely to seek immediate short term gain of lower value than a higher value gain that requires a longer wait, these findings occur both in financial and in addictive drug contexts. The question addressed by this paper is whether it is possible to develop a piece of software that engenders an intuitive understanding of the full impact or extent of a future financial gain though an interactive computer games based format.

The paper presents a proof of concept developed to present animated 3-D interactive graphical models of savings goals in a game-based format to engender a playable strategy and reward platform to enable the user to allocate a strategy and immediately see the consequences of their decision unfold in a time-accelerating display. In this way the software is intended to engender the frission of excitement delivered by such short term wait formats (e.g. roulette) but also deliver the immediacy of a reinforcement outcome [4]. Typical real-life savings rewards are about as distant as can be expected in life other than some health outcomes (e.g. mortgages and pensions have long term waits for a positive outcome). As banks, inevitably cut back on front-line staff, inevitably a reliance on software solutions to deliver savings consultancy will become prevalent. The current project was conceived as a mechanism for engaging users in a branch based context but without the need for a hands-on supervision. It could be seen as a pre-savings interview primer, or an interim educational intervention for clients who wish additional time to explore their options. The Microsoft SurfaceTM [5] has the potential to bring an element of coffee table to the high-street bank, as the banks try and break some of the more formal barriers between themselves and their customers. In particular, the Surface with its large interaction platform is perfect to exploit the concepts of 
embodied cognition [6, 7]. It allows customers to take control over their finances which engenders more customer confidence and ownership. The tool takes a customer centric approach and allows for a more personalised and bespoke approach to financial management with the added benefit of privacy and not having to feel pressured or embarrassed by a banking representative. As opposed to being bombarded with financial information in the form of text, customers can engage, through a user-friendly interface, with a more straightforward and visually informative package which has the potential to build a stronger relationship with both financial planning and the banking sector.

The crucial novelty of the application is to couple the use of pictures and gestures in the selection and allocation of financial choices. Images present a powerful way of creating a unique access point to knowledge in the brain, and coupling a normally abstract or text based notion of an expenditure item allows the pictorial information and scale of the item to enhance and enrich its representation in the brain of the user [6]. Furthermore, by coupling the allocation of expenditure to a physical gesture (perception and action), the impact of the decision is again embedded in the brain of the user in a more direct and also in a multi-modal way $[6,7,8]$. These two innovations combined with the game based, instant feedback model of the outcome can promote and engender learning and behaviour change. The challenge for many banks is to make their branches attractive places to customers with economic and lifestyle decisions to be made. Currently the average shopper is more likely to use a coffee shop or tea room to create breathing space to make a financial decision requiring finance (e.g. holiday, sofa, car, house). The absence of trained consultants in financial products in the average public space creates an opportunity for a Surface based solution to savings engagement.

\section{PLANNING AND SAVING}

\subsection{Deferring future gains}

The underlying economic problem is one of discounting of future gains. There are two models for the curve of future discount (exponential and hyperbolic [e.g., 9,10) but both are potentially addressable by a system that increases the salience and immediacy of the future gain[4]. This resulted in the current project, a working software package to demonstrate a playable application for roll-out in banks, building societies, citizens' advice bureau and other community outlets such as libraries.

The solution

To WPF[11]or not to WPF[12]? The current trend in surface projects is to shuffle and manipulate rectangular objects such as documents and photographs which allows rapid sorting and scaling of objects in a genuinely interactive and intuitive manner. Most development for Surface SDK appears to be done in WPF. So why choose XNA for development of this project? Whilst the "animation language" in WPF (called StoryBoard) is easier to learn XNA offers more control over 3D models. WPF as a whole is ideal for application interaction, rendering what the user is requesting. XNA is purpose built or high frame-rate 2D/3D rendering of objects with features like character animation and inverse kinematics. Table 1 shows a comparison of the key features of the two different platforms available for the Microsoft SDK. Both systems have their merits, but for the purposes developing an inviting and engaging interface the XNA platform was used to enable the drawing and subsequent animation of 3D objects.

Table 1. Comparison of pros and cons for delivery platform

\begin{tabular}{|c|c|c|c|}
\hline Platform & $\begin{array}{c}\text { Current } \\
\text { Use in } \\
\text { Surface } \\
\text { SDKT }\end{array}$ & $\begin{array}{c}\text { Availability of } \\
\text { demo-software }\end{array}$ & $\begin{array}{c}\text { Utility for } \\
\text { 3-D graphics } \\
\text { models }\end{array}$ \\
\hline XNA & Limited & Minimal & Excellent \\
\hline WPF & Ubiquitous & Good & $\begin{array}{c}\text { Best for 2-D } \\
\text { interaction }\end{array}$ \\
\hline
\end{tabular}

\subsection{Implementation}

Development was performed on Microsoft Surface SDK on PCs running Windows Vista. Final testing was performed on a modified Microsoft Surface, with additional clear Perspex surround for peripheral device interfaces. The design took account of contemporary concepts in multi-touch gestural interface design [13]. To maximise flexibility, we selected the minimum range of gestures from the user defined gesture set [14], relying on 'Drag' and the various 2point 'Enlarge(Shrink)' gestures, these gestures are easiest to port to other platforms and are already established currency in multi-touch devices such as smartphones.

\section{SOFTWARE DESIGN}

The game is presented in three screens characterize by three actions: Divide, Decide Spin (and Win). This allows the participant to allocate their resources, generate a savings plan and then play the game to see if their strategy works. If not, they can play again until they get the design they want. The top right panel of the layout screen shows the selection menu for income (green surround) and expenditure (red surround) items. Participants drag the item they want into the workspace panel, whereupon a selection of allocation-option satellite mini-icons pop 
up around the main icon to show the expenditure recurrence (e.g. weekly, monthly, annual etc.). The use of dragging and scaling[14] of pictorial items is crucial to the novelty of the application in the finance context, to associate perception and action $[6,7,8]$.

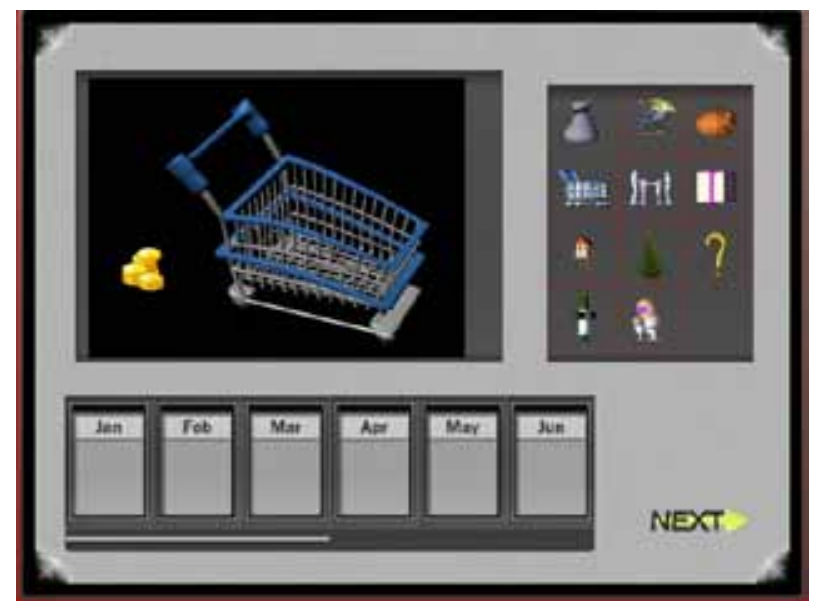

Figure 1. Layout of the decide section of the game where players must physically allocate their resources. Upper left panel indicates the resource allocation area where the pinch gesture is used to manipulate levels of expenditure and payment intervals. Upper right panel indicates the income and payment options, which can be selected and dragged to the allocation area. The lower panel displays the drag-scrollable 'gantt-chart' calendar for the income and expenditure schedule. Items allocated appear here once edited.

The initial strategy component of the game is to develop a portfolio of income expenditure corresponding to a desired spending plan for the participant. The scale of the expenditure can be adjusted by a pinch gesture revealing the scale of the expenditure in a digit echo readout above the panel. Figure 1 illustrates the look and feel of the initial interface, a multi-panel interface for the selection allocation and division of expenditure and income in an average consumer context.

Once the selections have been made, the participant can proceed to the savings stage where a series of doors are arranged to reveal the relative risk and return of each option. Again, using the pinch (or any two point scaling gesture) on the savings pot icon, the participant can select the amount or proportion of savings for each product they wish. Finally they can proceed to the final screen where they can 'Spin the wheel of time.' Savings plans ending in credit 'Win', those that end in debt, 'Lose'. Audio fanfares are played appropriate fanfares for each outcome. At this point the player is invited to revisit their choices and play again to achieve a better outcome. At the moment the default icons for playing are savings (illustrated by a piggy bank) and mortgages (illustrated by an animated house). In the future additional personalized savings goals will be playable.

\section{VISUAL APPEARANCE}

The icons were designed to be animated, and so are full 3-D models, imported to the project from MayaTM [15]. Each one was conceived by a concept artist and then translated into a 3-D model for export to the program.

By using icons instead of text headings the interface becomes more navigable and potentially of use to audiences without high levels of literacy or numeracy in the language of the host terminal. The purpose is to demonstrate universal principles of money use, so for multi-language societies, this demonstration minimizes the need for language rewrites. Concepts for characters were sketched in drawing pen, scanned scanned and printed off. Water colours were then used prior to scanning into Adobe PhotoshopTM. Individual character assets, for example arms, mouth and eyes, were drawn separately using the pen and brush tools and a WacomTM tablet. Once happy with the outline the artist would block colour-in all the assets and then make different shaders with gradients on every layer for each block colour. This was then used by the 3D modeller and technical artist to build the model in MayaTM. It was then textured and animated; each character has own movements appropriate for its context.

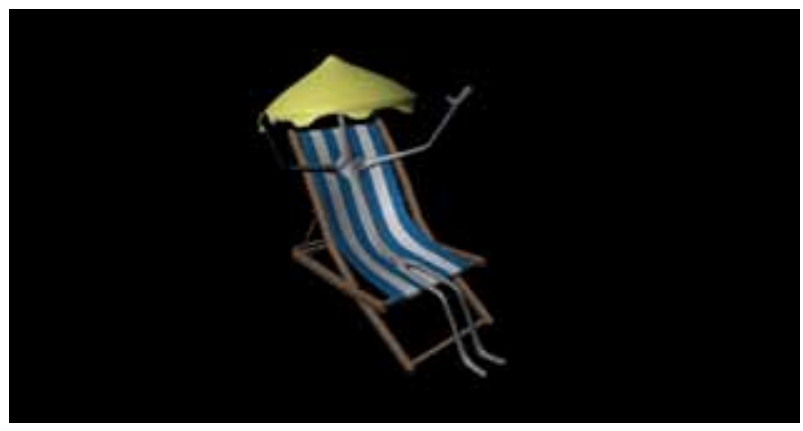

Figure 2. Sample icon for holiday holiday budget.

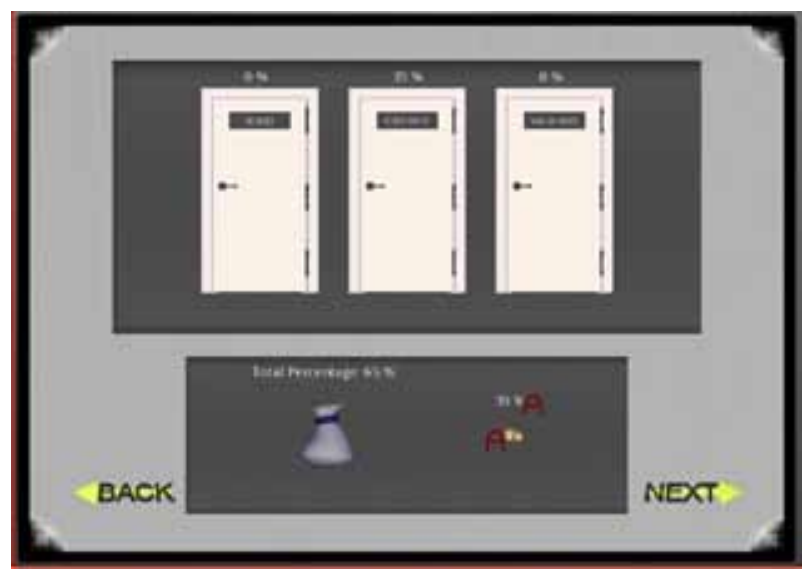

Figure 3. Screen layout for the decide section of the game where savings decisions are made and proportions allocated. 
The particular benefit of using the Microsoft Surface is the physical scale of the gestures required to make the allocations. The embodied cognition literature $[6,7,8]$ suggests that behavioural choices coupled with actions are more salient, and so the actually commitment to make an investment or gamble should be treated with a greater level of commitment than a simple mouse click or a keyboard entry.

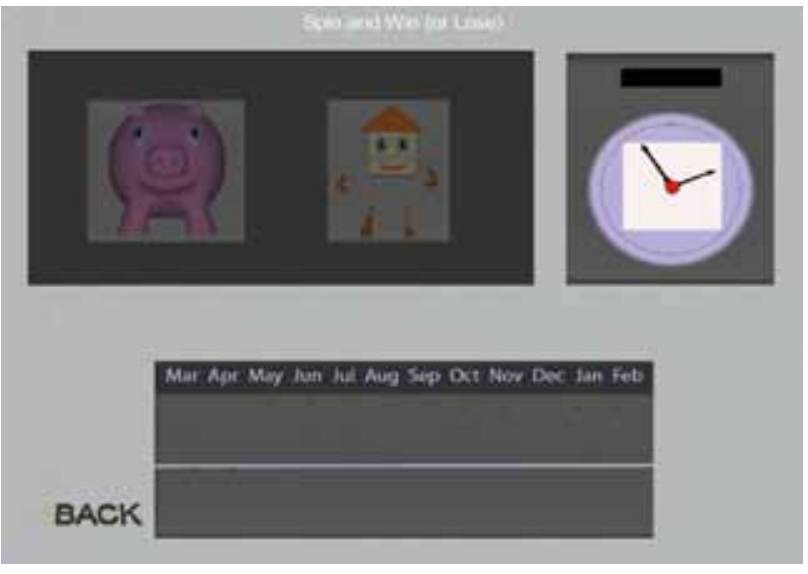

Figure 4. Screen layout for the spin and win (or lose) section of the game, the time wheel on the right panel can be spun by the user and stopped at a given point, as it spins savings or costs accrue.

\subsection{Demonstration Feedback}

Preliminary user feedback from informal demos has focused on two key areas, the most attractive aspect of the software solution was the removal of the need for participants to write down actual financial values (this appears to be a barrier to engagement in financial planning). The second key feature was the utility of the surface as an input device which was perceived to be novel, enjoyable and engaging. Initial developer feedback has focused on the development process for the software itself, in particular the facility to create additional expenditure items and the need for the program to focus on particular individual client narratives.

\section{SUMMARY AND FUTURE WORK}

A rapid prototype financial planning tool for Surface was developed by a multi-disciplinary team using the XNA platform to render 3-D graphical and modifiable icons. The innovation of the interface is focused on the incorporation of the concepts of embodied cognition to harness the linkage of the use of images with perception and action $[6,7,8,16]$. Response to testing has been positive but has highlighted the need for a focused and parallel strand of development to enable widespread development and adoption. The software is re-mappable to mobile devices and desktop PCs. The natural development of this 'time machine' concept is of multiple independent applications focused on individual purchasing decisions. For example a downloadable smartphone based application could help people decide the correct mobile tariff, or a college-fund app could help families determine a savings plan for a child to enable enrolment at a fee paying education institution. The possibilities for applications are extensive, but the underlying mathematical model of savings accrual can be re-used, as can the general interface. It is possible to re-skin the application interface to suit individual target markets.

The key to success is the context of the roll out platform. Ideally, for a Surface development pathway, this unit should be in a semi-structured situation where sales or debt counseling trained staff can be on hand to set up an interaction, allow free form play and then post-play to consult on future decisions. The beauty of the multi-touch interface and game format arise here - the format allows users to imagine scenarios and play at "what ifs'. In a formal bank sales consultancy scenario, where the user is immediately pinned down to their own financial situation the user can feel inhibited. A game-based more format allows for people not commit to the actual figures they possess. This can help reduce embarrassment, particularly for people who may have a problem, an experience such as this may be an easier way to make the transition to acknowledgement of a problem than an full on debt counseling session.

Ultimately, as multi-touch devices become prevalent in the home and the workplace the trend toward interactive input of data will emerge. In the meantime, this platform demonstrates the notion of deferred or discounted future gains to the player. The system can be used in a experimental situation to test the predictions of two different models of discounted future gains (the exponential and the hyperbolic models)[e.g., 9,10].

\section{ACKNOWLEDGMENTS}

This project was supported by NCR. Our grateful thanks to NCR and to Graham Johnson, Jonathan Black, Mark Grossi, Andrea Szymkowiak, Kate Ho, Paul Zealey, Paul Harris, Jim Bown, Louis Natanson, John Isaacs, Dave Heeley, Jim Moonie and colleagues from Abertay for helpful discussions and support in the development of this project.

\section{REFERENCES}

[1] Raineri, A., and Rachlin, H. 1993. The effect of temporal constraints on the value of money and other commodities. Journal of Behavioral DecisionMaking, 6, 77-94.

[2] Green, L., Fry, A. F., and Myerson, J. 1994. Discounting of delayed rewards: A life span 
comparison. Psychological Science, 5, 33-36.

[3] Kirby, K. N. 1997 Bidding on the future: Evidence against normative discounting of delayed rewards. Journal of Experimental Psychology: General 126, 54-70.

[4] Kalat, J. W, 2008 Introduction to Psychology, Thomson

[5] Microsoft Surface 2008 Microsoft Corporation http://www.microsoft.com/surface/en/us/default. aspx

[6] Barsalou, L.W. 2008 Grounding symbolic operations in the brain's modal systems. In G.R. Semin \& E.R. Smith (Eds.), Embodied grounding: Social, cognitive, affective, and neuroscientific approaches (pp. 9-42). New York: Cambridge University Press.

[7] Barsalou, L.W. 2008 Grounded cognition. Annual Review of Psychology, 59, 617-645.

[8] Clark A. 1997. Being There: Putting Brain, Body, and World Together Again. Cambridge, MA: MIT Press

[9] Vuchinich, R. E., and Simpson, C. A. 1998. Hyperbolic temporal discounting in social drinkers and problem drinkers. Experimental and Clinical Psychopharmacology, 6, 292-305.

[10] Bickel, W. K., \& Johnson, M. W. 2003. Delay discounting: A fundamental behavioral process of drug dependence. In G. Loewenstein, D. Read \& R. F. Baumeister (Eds.), Time and Decision. New York: Russell Sage Foundation.

[11] WPF. 2010 Microsoft Corporation http:// windowsclient.net/

[12] XNA. 2007 Microsoft Corporation. http://www. xna.com/

[13] Saffer, D., 2009. Designing gestural interfaces. O'Reilly.

[14] Wobbrock, J.O., Morris, M.R. and Wilson, A.D. 2009. User-defined gestures for surface computing. Proceedings of the ACM Conference on Human Factors in Computing Systems (CHI '09). Boston, Massachusetts (April 4-9, 2009). New York: ACM Press, pp. 1083-1092.

[15] Maya. 2008 Autodesk Maya. http://usa. autodesk.com/

[16] Jostmann, N. B., Lakens, D., \& Schubert,T. W. (2009). Weight as an embodiment of importance. Psychological Science, 20, 1169-1174. 